\title{
A search for water masers toward extrasolar planets
}

\author{
V. Minier ${ }^{1,2,3}$ and C. Lineweaver ${ }^{3,4}$ \\ 1 Service d'Astrophysique, DAPNIA/DSM/CEA Saclay, 91191 Gif-sur-Yvette, France \\ e-mail: vincent.minier@cea.fr \\ 2 AIM, Unité Mixte de Recherche, CEA-CNRS-Université Paris VII, UMR 7158, CEA/Saclay, 91191 Gif-sur-Yvette, France \\ 3 School of Physics, University of New South Wales, Sydney NSW 2052, Australia \\ 4 Planetary Science Institute RSAA/RSES, Australian National University, Canberra ACT 0200, Australia
}

Received 1 March 2005 / Accepted 2 December 2005

\section{ABSTRACT}

Context. Water is the most common triatomic molecule in the universe and the basis of life on Earth. Astrophysical masers have been widely studied in recent years and have been shown to be invaluable probes of the details of the environment in which they are found. Water masers, for instance, are often detected toward low-mass star-forming regions. Doppler radial-velocity surveys have detected about 160 exoplanets. Aims. Observations of water masers from exoplanetary systems would give us a new detailed window through which to explore them. Methods. We present a search for water masers toward eighteen extrasolar planets using the newly upgraded Australia Telescope Compact Array at $12 \mathrm{~mm}$. A sensitivity of $\sim 25 \mathrm{mJy}^{\text {beam }}{ }^{-1}$ and an angular resolution of $\sim 10^{\prime \prime}$ were achieved at $22.235 \mathrm{GHz}$.

Results. No maser lines are clearly observed.

Key words. masers - planetary systems - circumstellar matter

\section{Introduction}

Water is the most common triatomic molecule in the Universe and the basis of life on Earth. Doppler radial-velocity surveys have detected about 160 planets $^{1}$ orbiting nearby solar-type stars including 18 multiple planet systems. The proximity and availability of water on or near these exoplanets is an important piece of our emerging picture of how our Solar System compares to these newly detected planetary systems, and more speculatively what the prospects for water-based life are.

Cosmic thermal water emission is not easily detectable with ground-based telescopes because the large amount of vapour in the lower atmosphere of the Earth contributes to the emission itself and absorbs radiations in the sub-millimetre and infrared parts of the electromagnetic spectrum, where water spectral lines are largely present (e.g. Boonman et al. 2003; Deguchi \& Nguyen-Q-Rieu 1990). However, non-thermal water emission is observed from Earth: water vapour masers (e.g. Cheung et al. 1969; Waters et al. 1980) are very intense and widespread cosmic phenomena that have been detected toward star-forming regions (Genzel \& Downes 1979), late M-type stars (Dickinson 1976) and Active Galactic Nuclei (Claussen et al. 1984). They were detected at $22.235 \mathrm{GHz}$ (Cheung et al. 1969), $183.309 \mathrm{GHz}$ (Cernicharo et al. 1990), $321 \mathrm{GHz}$ and $325 \mathrm{GHz}$ (Menten et al. 1990a,b).

Recently, detections of various types of water emission in the Solar System have been reported. Water abundances

\footnotetext{
1 As reported in http://www. obspm. fr/planets in June 2005.
}

were measured in the atmosphere of giant planets with the $I S O$ and SWAS space observatories (e.g. Bergin et al. 2000) and in comets with the ODIN satellite (Lecacheux et al. 2003). Thermal water emission and $22-\mathrm{GHz}$ water masers were also seen coming from the impact on Jupiter induced by the Shoemaker-Levy comet collision (Bjoraker et al. 1996; Cosmovici et al. 1996). Finally, water absorption features were identified toward the sunspot umbrae and might originate on the Sun (Wallace et al. 1995).

Traces of water emission have also been reported near solar-type star systems. Water masers were for instance imaged in NGC 2071 where they might trace a protoplanetary disk around a $1-M_{\odot}$ protostar (Torrelles et al. 1998). Evidence for water emission was discovered toward early M-type giant stars (Tsuji et al. 1997). Water masers also arise in the circumstellar envelopes of more evolved giant stars such as Mira and AGB stars (e.g. Takaba et al. 2001) and continue to glare in post-AGB stars and young planetary nebulae (e.g. Engels 2002). This demonstrates that $\mathrm{H}_{2} \mathrm{O}$ can be present in the environment of a wide range of stars in the HR diagram.

In 2002, possible detections of water masers toward extrasolar planets were presented by Cosmovici et al. (2002) during the Second European Workshop on Exo/Astrobiology in Graz, Austria although the results have remained unpublished ${ }^{2}$. The maser lines were possibly detected toward Epsilon Eridani,

2 This possible discovery was announced through New Scientist $175(2361), 22$. 
Upsilon Andromedae and 47 Ursa Majoris at a level of a few $100 \mathrm{mJy}$ (Cosmovici, priv. comm.). However, null results have also been announced for five exoplanet host stars down to a sensitivity of 14-64 mJy (Greenhill, IAU circular 7985), of which Upsilon Andromedae and Epsilon Eridani had been reported to emit a possible water maser. Non-detections were also reported by Butler et al. (2002) after observing Epsilon Eridani, Upsilon Andromedae and 47 Ursa Majoris down to a sensitivity of 2.6-10.2 mJy with the VLA.

In this letter, we present the results of a search for water masers toward 18 extrasolar planets. The objective was to study whether water masers can form in the near environment (e.g. planetary atmosphere, circumstellar environment or comet clouds) of extrasolar planets. The targets were selected to be observable from the Southern latitudes out of $\sim 150$ known exoplanets available at the date of the observations. The high sensitivity and high angular resolution search was conducted with the Australia Telescope Compact Array, that was upgraded in April 2003 to operate at $12 \mathrm{~mm}$. Observations of water masers from exoplanetary systems would give us a new detailed window through which to explore them. Details extractable from maser detection include answers to the following questions: what is the velocity of the masing source (e.g. planets or comets)? Which part of the planetary system is compatible with the column densities of water, a pumping mechanism and a lack of collisional thermalization that would otherwise quench the maser? By combining observations and models, the physical conditions of the exoplanetary systems may be probed.

\section{Conditions for detecting water masers toward extrasolar planets}

The mechanisms that might generate $22-\mathrm{GHz}$ water maser emission in extrasolar planets include (but are not limited to) cometary impacts in atmospheres of giant planets, particularly in younger stellar systems in which much more massive and frequent impacts are expected (e.g. Chyba 1990; Shoemaker 1983). Whether the required conditions (column density, temperature, velocity coherence, path length, pumping) are present to produce detectable water masers is unknown.

Water maser action needs a large column density of water vapour and a pumping mechanism to ensure the inversion of the population levels $6_{16}-5_{23} .22-\mathrm{GHz}$ masers are probably pumped by collisions with $\mathrm{H}_{2}$, although radiative pumping is also proposed to explain masers at higher frequencies (Yates et al. 1997). Interstellar and stellar 22-GHz masers require relatively high $\mathrm{H}_{2}\left(<10^{8}-10^{10} \mathrm{~cm}^{-3}\right)$ and water $\left(10^{3}-10^{5} \mathrm{~cm}^{-3}\right)$ densities, but with an abundance ratio to $\mathrm{H}_{2}<10^{-4}$, and kinetic temperatures within 200-2000 K (Yates et al. 1997).

Jupiter-like planets whose atmosphere contains $\sim 80 \%$ of $\mathrm{H}_{2}$, might offer suitable conditions for water masers, assuming that a large amount of water vapour is present. In Jupiter, the water vapour appears to lie at high atmospheric altitudes in the middle stratosphere (Bergin et al. 2000, and references therein). The $\mathrm{H}_{2} \mathrm{O}$ abundance results from both internal chemistry and external transport through the Shoemaker-Levy 9 comet, and is stable against photolysis and conversion to $\mathrm{CO}_{2}$ over typically 50 years (Lellouch et al. 2002). The average physical conditions for the bulk of water vapour at $p<$ 5.5 mbar are $T=150 \mathrm{~K}, N\left(\mathrm{H}_{2} \mathrm{O}\right)=2.8 \times 10^{15} \mathrm{~cm}^{-2}$ and the $\mathrm{H}_{2} \mathrm{O}$ abundance ratio to $\mathrm{H}_{2} \sim 10^{-9}$. In the case of a deep impact, a large amount of water vapour can be detected in the hot upper atmosphere (Bjoraker et al. 1996) where $p=3 \mu$ bar and $T>200 \mathrm{~K}$ (Young 2003). The $\mathrm{H}_{2} \mathrm{O}$ abundance ratio to $\mathrm{H}_{2}$ increases to $10^{-7}$ with higher altitudes (Fig. 2 in Bergin et al. 2000).

Another vital parameter for maser action is the pumping efficiency. Collisional pumping with $\mathrm{H}_{2}$ molecules might occur in the hot upper atmosphere and deeper in the stratosphere, where the temperature could increase to $>200 \mathrm{~K}$ in shocked gas. These elements suggest that suitable conditions for planetary water masers are probably met in Jupiter-like planet atmospheres following a comet impact. The existence of water masers in Earth-like planets is less likely because the Earth atmosphere is characterised by a large column density of thermalised water vapour.

Outside the Solar System, the collision of relatively large comets with a planet could enhance the water vapour abundance and induce maser action if the pumping and the velocity coherence are sufficiently effective along the path length. To estimate the physical conditions that produce masers with a peak intensity $\sim 100 \mathrm{mJy}$, we consider the case of a comet impact that would inject water vapour in the atmosphere of a Jupiter-like planet (140000 km in diameter). In this simplified model, the masing path length is the thickness of the atmosphere in which water vapour is present. It can vary between $300 \mathrm{~km}$ (a stratosphere path length) and $\sim 2 \times 10^{4} \mathrm{~km}$ (the maximum tangential path length for a $1000-\mathrm{km}$ atmosphere). A single tangential path length of $1.6 \times 10^{4} \mathrm{~km}$ is adopted for the purpose of this work. It corresponds to the maximum path length for a height of $500 \mathrm{~km}$ in the atmosphere. The maser amplifies a background radiation with a brightness temperature corresponding to the local atmospheric temperature. Two kinetic $\mathrm{H}_{2}$ temperature $\left(T_{\mathrm{H}_{2}}\right)$ cases are studied: $150 \mathrm{~K}$ (stratosphere) and $300 \mathrm{~K}$ (hot upper atmosphere or shocked gas). The unsaturated maser brightness temperature is given by $T_{\mathrm{H}_{2}} \mathrm{e}^{\tau}$, where $\tau$ is the maser gain coefficient. The value of $\tau$ is, in first approximation, proportional to the path length, the velocity distribution function $\left(1 / \Delta v_{\mathrm{D}}\right.$ at the line centre, where $\Delta v_{\mathrm{D}}$ is the thermal doppler broadening) and the population difference $\left(n_{1}-n_{2}\right.$, where $n_{1}$ and $n_{2}$ are the population of the lower and upper levels, respectively). Once $\tau$ is known for a given flux density translated in brightness temperature, one can deduce the total column density of water vapour by assuming that the pumping efficiency $\left(n_{1}-n_{2} / n_{1}+n_{2}\right)$ is $\sim 1 \%$ and $n_{1}$ nearly obeys the Boltzmann distribution.

Table 1 presents the physical conditions required to produce a $100-\mathrm{mJy}$ maser at distances of $10-100 \mathrm{pc}$. The resulting $\mathrm{H}_{2} \mathrm{O}$ density varies between $10^{10}$ and $10^{11} \mathrm{~cm}^{-3}$ depending on $T_{\mathrm{H}_{2}}$. These values do not satisfy the density conditions and longer path lengths $\left(\gg 10^{6} \mathrm{~km}\right)$ are desirable to avoid high density that will quench the inversion population. If the maser occurs in the planetary atmosphere, its $\mathrm{H}_{2} \mathrm{O}$ column density has to be enhanced by a factor $10^{3}$ to $10^{5}$ to allow maser action assuming initial conditions comparable to those in Jupiter (see above). The $\mathrm{H}_{2} \mathrm{O}$ abundance ratio to $\mathrm{H}_{2}$ varies between 
Table 1. Physical conditions to produce a 100-mJy Jovian water maser at a distance $D$. Standard maser theory is used (Reid \& Moran 1988). $R$ is the radius of the comet nucleus and $M_{\mathrm{H}_{2} \mathrm{O}}$ is the total mass of water in it. A mean ice density of $300 \mathrm{~kg} \mathrm{~m}^{-3}$ is used for the comet nucleus. $N_{\mathrm{H}_{2} \mathrm{O}}$ is the column density in the planet atmosphere.

\begin{tabular}{lllll}
\hline \hline$T_{\mathrm{H}_{2}}(\mathrm{~K})$ & 150 & 150 & 300 & 300 \\
$D(\mathrm{pc})$ & 10 & 100 & 10 & 100 \\
\hline$N_{\mathrm{H}_{2} \mathrm{O}}\left(10^{19} \mathrm{~cm}^{-2}\right)$ & 6.9 & 8.6 & 3.2 & 4.0 \\
$R(\mathrm{~km})$ & 6.3 & 6.8 & 4.9 & 5.2 \\
$M_{\mathrm{H}_{2} \mathrm{O}}\left(10^{14} \mathrm{~kg}\right)$ & 3.2 & 4.0 & 1.4 & 1.8 \\
\hline
\end{tabular}

$10^{-6}$ and $10^{-4}$. A large amount of water ice, $10^{14}-10^{15} \mathrm{~kg}$, is needed to fill in part of the planet atmosphere (Table 1). In comparison, Bjoraker et al. (1996) measured a column density of $10^{18} \mathrm{~cm}^{-2}$ and a total water mass of $10^{9} \mathrm{~kg}$ toward the impacts of the Shoemaker-Levy 9 comet.

In summary, an extrasolar planet must undergo a much more severe bombardment than Jupiter in 1994 to produce a detectable water maser at a level of $100 \mathrm{mJy}$. However, the presence of 100-mJy masers is difficult to explain in a planetary environment in terms of standard $\mathrm{H}_{2} \mathrm{O}$ maser theory (e.g. Reid \& Moran 1988). Elitzur et al. (1989) have characterised the maser emission measure with a parameter $\xi$ (see Eq. (2.1) in Elitzur et al.), which is directly proportional to the water vapour abundance ratio to $\mathrm{H}_{2}$, the square of the $\mathrm{H}_{2}$ density and the path length. To compensate the relatively short planetary path length and obtain $\xi>1$, either large $\mathrm{H}_{2} \mathrm{O}$ abundance ratio or large gas density is required, which might be unrealistic in the first case and could quench the maser in the second case. Finally, the velocity coherence is implicitely assumed to be reached along the full path length, which is unlikely to occur along a sufficiently large path length in such a turbulent atmosphere.

\section{Observations}

Interferometers allow both high angular resolution and high sensitivity observations. These technical characteristics are ideal in the search for water masers toward extrasolar planets. A small beam could clearly establish the connection between water masers and exoplanets in case of detection. Furthermore, masers from a planetary atmosphere are probably highly variable as the planet rotates (only 10-h sidereal period for Jovian planets).

In April 2003, we used the newly upgraded ATCA system at $12 \mathrm{~mm}$ in the EW352-baseline configuration plus antenna 6 . Eighteen extrasolar planets were searched for $22-\mathrm{GHz}$ water masers down to a noise rms of about $25 \mathrm{mJy}^{\text {beam }}{ }^{-1}$ (average weather conditions), excluding antenna 6 in the rms estimate. A bandwidth of $16 \mathrm{MHz}$ and 512 spectral channels were used, which give a spectral resolution of $31 \mathrm{kHz}$ or $0.4 \mathrm{~km} \mathrm{~s}^{-1}$ and a total velocity range of $216 \mathrm{~km} \mathrm{~s}^{-1}$ centred at $V_{\mathrm{lsr}}=0 \mathrm{~km} \mathrm{~s}^{-1}$. An angular resolution of about $10 \operatorname{arcsec}$ was obtained with the EW352 configuration, allowing us to probe the inner $~ 30-1200 \mathrm{AU}$ of the planetary systems depending on their distance to us. Each target was observed in a straight 30 -min scan in average, preceded and followed by a 3-min scan on the phase calibrator. The high sensitivity of ATCA at $12 \mathrm{~mm}$ allows us to reach a $4-\sigma \mathrm{rms}$ of $100 \mathrm{mJy}^{\text {beam }}{ }^{-1}$ in 30 minutes, which is $1 / 20$ of the Jupiter sidereal period.

\section{Data analysis, results and discussion}

The data were analysed with Miriad in an essentially identical method as for the centimetre data analysis (Sault \& Killeen 2004). The amplitude calibration was achieved on Uranus. The bandpass solutions were obtained with a strong calibrator, 1730-130. Pointing corrections were estimated and applied every 2 hours in average. The phase calibration was made with secondary calibrators. A couple of well known maser sources were successfully observed during the experiment to check the new 12-mm system.

Vector-averaged cross-correlated spectra were generated from the data cubes for each extrasolar planet. All baselineaveraged spectra were first inspected.

The individual baseline spectra were also averaged over various time ranges $(\sim 2-20 \mathrm{~min})$ and searched for artificial lines and peaks. Possible maser lines from a rotating planetary atmosphere could then be monitored in velocity as long as the maser was bright enough to be identified in a few minutes. Conversely, non detection could be the result of signal dilution in velocity/channels over a significative fraction of sidereal period. If a signal was visualised, the spectrum was Hanning smoothed and checked again. Both polarisation spectra were also inspected in a similar fashion. The $1-\sigma \mathrm{rms}$ on the noise baseline varies between 24 and $31 \mathrm{mJy}$ for our source sample (Table 2). This includes the noise bias value, which is a residual noise level obtained after vector averaging. After all the above checks, a signal was considered as a possible detection when the flux intensity was greater than $\sim 4 \sigma$ and the line was still visible after Hanning smoothing.

The vector-averaged spectrum for HD 47536, a KIII giant star with a giant planet candidate (Setiawan et al. 2003), exhibits a noise level greater than $\sim 3-4 \sigma$ in a few channels. The intensity level reaches $100 \mathrm{mJy}$ around $V_{\mathrm{lsr}}=-36 \mathrm{~km} \mathrm{~s}^{-1}$. Channel maps were produced after averaging over 7 channels $\left(3 \mathrm{~km} \mathrm{~s}^{-1}\right)$ that corresponds to the $F W H M$ of the putative maser line. A 100-mJy signal was identified in the channel map at the averaged velocity of $-36 \mathrm{~km} \mathrm{~s}^{-1}$. However, a few channel maps exhibit a flux density level up to $50 \mathrm{mJy}$. It is thus possible that the emission at $-36 \mathrm{~km} \mathrm{~s}^{-1}$ is associated with a noise peak rather than a true signal. New ATCA observations in 2004 failed to confirm this possible detection.

Finally, no maser is observed toward Epsilon Eridani, a target that was reported to exhibit a possible maser line (Cosmovici et al. 2002). This result confirms other nondetection reports by Greenhill (IAU circular 7985) and Butler et al. (2002). Our sensitivity limit for Epsilon Eridani was $26 \mathrm{mJy}$ beam $^{-1}$. This value compares well with those achieved by other instruments such as the VLA and large aperture single dish telescope. The possible signal toward Eps Eri was measured at an intensity level $>3 \sigma$ (with $1 \sigma=200 \mathrm{mJy}$ ) with the Medicina 32-m radio telescope (Cosmovici, priv. comm.). The ATCA non-detection results suggest that the lines observed by Cosmovici et al. are (i) transient masers, (ii) signals from 
Table 2. Exoplanet sample and results. The age of each star is estimated with $\log (t)=10.725-1.334 \times R+0.4085 \times R^{2}-0.0522 \times R^{3}($ Donahue 1993), where $R$ is the chromospheric activity times $10^{5}$.

\begin{tabular}{|c|c|c|c|c|c|c|c|c|c|}
\hline \multirow{2}{*}{$\begin{array}{l}\text { HD } \\
\text { name }\end{array}$} & \multirow{2}{*}{$\begin{array}{l}\text { Spectral } \\
\text { type }\end{array}$} & \multirow{2}{*}{$\begin{array}{l}\text { Mass } \\
\left(M_{\odot}\right)\end{array}$} & \multirow{2}{*}{$\begin{array}{l}\text { Distance } \\
(\mathrm{pc})\end{array}$} & \multirow{2}{*}{$\begin{array}{l}\text { Age } \\
\left(10^{9} \mathrm{yr}\right)\end{array}$} & \multicolumn{2}{|c|}{ Coordinates } & \multirow{2}{*}{$\begin{array}{l}\text { Radial vel. } \\
\left(\mathrm{km} \mathrm{s}^{-1}\right)\end{array}$} & \multirow{2}{*}{$\begin{array}{l}\mathrm{rms} \\
(\mathrm{mJy})\end{array}$} & \multirow[t]{2}{*}{ Detection } \\
\hline & & & & & RA(J2000) & $\operatorname{Dec}(\mathbf{J} 2000)$ & & & \\
\hline HD 169830 & F8V & 1.40 & 36.32 & 4.3 & $18: 27: 49.5$ & $\begin{array}{l}-29: 49: 00 \\
\end{array}$ & $?$ & 28 & No \\
\hline HD 179949 & F8V & 1.24 & 27.05 & 2.0 & $19: 15: 33.2$ & $-24: 10: 45$ & -25.5 & 24 & No \\
\hline HD 202206 & G6V & 1.15 & 46.34 & $?$ & $21: 14: 57.8$ & $-20: 47: 21$ & 14.7 & 26 & No \\
\hline HD 213240 & G4IV & 1.22 & 40.75 & 2.7 & $22: 31: 00.4$ & $-49: 25: 59$ & -0.9 & 26 & No \\
\hline HD $216435^{1}$ & G0V & 1.25 & 33.29 & 5.6 & $22: 53: 37.9$ & $-48: 35: 53$ & -1.0 & 26 & No \\
\hline HD 142 & G1IV & 1.10 & 25.64 & 4.2 & $00: 06: 19.2$ & $-49: 04: 30$ & 2.6 & 25 & No \\
\hline HD 2039 & G2.5IV-V & 0.98 & 89.85 & 4.0 & $00: 24: 20.3$ & $-56: 39: 00$ & $?$ & 27 & No \\
\hline HD 6434 & G3V & 1.00 & 40.32 & 3.7 & 01:04:40.2 & $-39: 29: 17$ & 22.4 & 27 & No \\
\hline HD 13445 & K0V & 0.80 & 10.91 & 2.2 & 02:10:25.9 & $-50: 49: 25$ & 53.1 & 26 & No \\
\hline HD $17051^{2}$ & G0V & 1.03 & 17.24 & 1.6 & $02: 42: 33.5$ & $-50: 48: 01$ & 15.0 & 25 & No \\
\hline HD $22049^{3}$ & $\mathrm{~K} 2 \mathrm{~V}$ & 0.80 & 3.22 & 0.7 & $03: 32: 55.8$ & $-09: 27: 29$ & 15.5 & 26 & No \\
\hline HD 23079 & F9V & 1.10 & 34.60 & 4.8 & 03:39:43.1 & $-52: 54: 57$ & $?$ & 29 & No \\
\hline HD $27442^{4}$ & K2IV & 1.20 & 18.23 & $?$ & $04: 16: 29.0$ & $-59: 18: 07$ & 29.3 & 29 & No \\
\hline HD 30177 & G8V & 0.95 & 54.71 & 7.4 & $04: 41: 54.4$ & $-58: 01: 14$ & $?$ & 30 & No \\
\hline HD 47536 & K0III & 1.10 & 121.36 & $>5$ & $06: 37: 47.6$ & $-32: 20: 23$ & 78.8 & 31 & No? \\
\hline HD 73526 & G7V & 1.02 & 94.61 & $?$ & $08: 37: 16.5$ & $-41: 19: 08$ & $?$ & 29 & No \\
\hline HD 75289 & G0V & 1.15 & 28.94 & 4.9 & $08: 47: 40.4$ & $-41: 44: 12$ & 14.0 & 27 & No \\
\hline HD 83443 & K0V & 0.79 & 43.54 & 3.2 & 09:37:11.8 & $-43: 16: 19$ & 27.6 & 29 & No \\
\hline
\end{tabular}

Notes: ${ }^{1}$ Taul Gru; ${ }^{2}$ Iot Hor; ${ }^{3}$ Eps Eri; ${ }^{4}$ Eps Ret.

another location in the sky that was detected in the large beam $\left(\sim 1.5^{\prime}\right)$ of the Medicina 32-m telescope or (iii) artificial lines (e.g. RFI).

In conclusion, our observations have confirmed that detectable water masers in planetary systems are rare phenomena. Whether these phenomena can occur in an exoplanetary environment is an issue that can only be solved with very highsensitivity radio telescopes. Our results and conclusions are comparable to an unsuccessful search for cyclotron masers toward extrasolar systems (Bastian et al. 2000). Randomly distributed observations in time with large aperture radio instruments (e.g. SKA in the future) might possibly be useful to further address this issue.

Acknowledgements. The Australia Telescope Compact Array is part of the Australia Telescope which is funded by the Commonwealth of Australia for operation as a National Facility managed by CSIRO. We thank Ray Norris, Bob Sault and Cormac Purcell for their help in the ATCA observations.

\section{References}

Bastian, T. S., Dulk, G. A., \& Leblanc, Y. 2000, ApJ, 545, 1058

Bergin, E. A., Lellouch, E., Harwit, M., et al. 2000, ApJ, 539, L147

Bjoraker, G. L., Stolovy, S. R., Herter, T. L., Gull, G. E., \& Pirger, B. E. 1996, Icarus, 121, 411

Boonman, A. M. S., Doty, S. D., van Dishoeck, E. F., et al. 2003, A\&A, 406, 937

Brown, J. A., Johnson, H. R., Cutright, L. C., Alexander, D. R., \& Sharp, C. M. 1989, ApJS, 71, 623

Butler, B. J., Chandler, C. J., Claussen, M. J., \& Greenhill, L. J. 2002, A\&AS, 201, 4612

Cernicharo, J., Thum, C., Hein, H., et al. 1990, A\&A, 231, 15

Cheung, A. C., Cudaback, D. D., Rank, D. M., et al. 1969, BAAS, 1, 236
Chyba, C. F. 1990, Nature, 343, 129

Claussen, M. J., Heiligman, G. M., \& Lo, K. Y. 1984, Nature, 310, 298

Cosmovici, C. B., Montebugnoli, S., Orfei, A. Pogrebenko, S., \& Colom, P. 1996, P\&SS, 44, 735

Cosmovici, C., Teodorani, M., Montebugnoli, S., \& Maccaferri, G. 2002, presentation at the Second European Workshop on Exo/Astrobiology, Graz, Austria

Deguchi, S., \& Nguyen-Q-Rieu 1990, ApJ, 360, 27

Dickinson, D. F. 1976, ApJS, 30, 259

Donahue, R. A. 1993, Ph.D. Thesis, New Mexico State University

Elitzur, M., Hollenbach, D. J., \& McKee, C. F. 1989, ApJ, 346, 983

Engels, D. 2002, A\&A, 388, 252

Genzel, R., \& Downes, D. 1979, A\&A, 72, 234

Lecacheux, A., Biver, N., Crovisier, J., et al. 2003, A\&A, 402, 55

Lellouch, E., Bézard, B., Moses, J. I., et al. 2002, Icar, 159, 112

Menten, K. M., Melnick, G. J., \& Phillips, T. G. 1990a, ApJ, 350, 41

Menten, K. M., Melnick, G. J., Phillips, T. G., \& Neufeld, D. A. 1990b, ApJ, 363, 27

Reid, M. J., \& Moran, J. M. 1998, in Galactic and extragalactic radio astronomy, 2nd edn. (Berlin and New York, Springer-Verlag), 255

Sault, B., \& Killeen, N. 2004, The Miriad User Guide, http://www . atnf.csiro.au/computing/software/miriad/

Setiawan, J., Hatzes, A. P., von der Luhe, O., et al. 2003, A\&A, 398, L19

Shoemaker, E. M. 1983, AREPS, 11, 461

Takaba, H., Iwate, T., Miyaji, T., \& Deguchi, S. 2001, PASJ, 53, 517

Torrelles, J. M., Gómez, J. F., Rodríguez, L. F., et al. 1998, ApJ, 505, 756

Tsuji, T., Ohnaka, K., Aoki, W., \& Yamamura, I. 1997, A\&A, 320, 1

Wallace, L., Bernath, P., Livingston, W., et al. 1995, Science, 268, 1155

Waters, J. W., Kakar, R. K., Kuiper, T. B. H., et al. 1980, ApJ, 235, 57

Yates, J. A., Field, D., \& Gray, M. D. 1997, MNRAS, 285, 303

Young, R. E. 2003, NewAR, 47, 1 